\title{
Hypothyroidism with too Many Associations
}

\author{
Razzak $\mathrm{MA}^{1}$, Zia FB ${ }^{2}$ \\ DOI: https://doi.org/10.3329/jafmc.v17i1.56722
}

\begin{abstract}
Hypothyroidism is one of the common endocrine disorders. Amongst other causes, autoimmune hypothyroidism is clinically more important. It has got many adverse effects on metabolism, cardiovascular, musculoskeletal, fetomaternal and mental health. It's associated with obesity, dyslipidemia, IHD, inflammatory myopathy, depression etc. It is responsible for menorrhagia, subfertility, recurrent abortion, prematurity, fetomaternal morbidity and mortality. It may be associated with other organ specific or multisystem autoimmune diseases like SLE, vitiligo, alopecia, type-I DM, pernicious anemia and others. We have diagnosed a case of autoimmune hypothyroidism who had all the classic features of hypothyroidism. He had low FT4 and very high TSH. Autoimmunity was confirmed by high titer of anti TG and anti TPO antibody. In addition he had dimorphic anaemia, alopecia, diastolic hypertension, dyslipidemia, IHD, Vit D deficiency, myopathy, peripheral neuropathy, depression and dementia. His condition has been improved with Thyroxin replacement, lipid lowring drugs, anti anginal drugs, iron, calcium, folic acid, Vit-D supplementations, exercise, physiotherapy and other symptomatic and supportive measures. He was advised for consultation with dermatologist and periodic follow up.
\end{abstract}

Key-words: Hypothyroidism, Autoimmune, Alopecia universalis, Myopathy, Dyslipidemia, IHD.

\section{Introduction}

Hypothyroidism, also called inactive thyroid, is a disorder of endocrine system, in which the thyroid gland does not produce enough thyroid hormone ${ }^{1}$. Common causes are lodine deficiency, Hashimoto's thyroiditis, thyroidectomy, radioiodine therapy in Graves' disease, neck radiation and drug induced ${ }^{1}$. Usual manifestations are cold intolerance, weight gain in spite of poor appetite, tiredness, constipation, change of voice, sleepiness, depression etc ${ }^{1}$. In female, there may be menorrhagia, subfertility, recurrent abortion and other fetomaternal complications. In a male it may cause erectile dysfunction. If diagnosis and treatment is delayed, it may produce life threatening and disabling complications like myxedema coma, madness, diastolic hypertension, Pericardial effusion, dyslipidemia, IHD, cardiomyopathy, heart failure, myopathy, peripheral and entrapment neuropathy, rhabdomyolysis, (acute kidney injury) AKI, obstructive sleep apnea and (Nonalcoholic fatty liver disease) NAFLD ${ }^{1}$. Autoimmune hypothyroidism may be associated with other autoimmune diseases like vitiligo, alopecia, SLE, polymyositis, pernicious anemia, Type-I DM etc². Hypothyroidism has got link with Type-II DM and Metabolic syndrome as well. We have diagnosed a case of autoimmune hypothyroidism in August 2019. The patient presented with too many associations and complications. We are publishing the case report to generate awareness amongst all concerned about the diverse clinical manifestations and various associations of Hypothyroidism.

\section{Case Report}

A 35-years-old male patient presented to a private chamber in August-2019 with the history of progressive weight gain in spite of poor appetite, increased sleep, constipation, cold intolerance and hoarseness of voice for last few years. He also noticed gradual loss of scalp and body hairs including beard and eyebrows for same duration. He developed body ache, myalgia and weakness of proximal muscles of the limbs and occasional exertional compressive central chest pain for last 3 months. He had general weakness, fatigability, slowness of activities and forgetfulness. There was no past history of hypertension, diabetes mellitus, ischemic heart disease, bronchial asthma or any rheumatologic diseases. Clinically he was pale, puffy, depressed and dull looking (Fig-1). He had crocky monotonous voice, non pitting edema and dry skin. There was no thyromegaly, lymphadenopathy, skin rash or arthritis. There was total absence of hairs in scalp, face and other parts of the body (Fig-1). His pulse was $60 / \mathrm{min}$. Blood pressure (BP):130/100 mm Hg. Examination of precordium, chest and abdomen revealed no abnormality. His cranial nerves were intact and there was no ptosis. He had significant weakness (Grade III $/ \mathrm{V}$ ) of proximal muscles of the limbs with mild impairment of sensation and delayed ankle jerks. Other jerks were grossly diminished. Provisionally he was diagnosed as a case of Hypothyroidism with diastolic hypertension, Ischemic heart diseases, Myopathy, and Alopecia Universalis. He was extensively investigated to confirm the diagnosis and associated conditions and to rule out the other possibilities. His CBC revealed Hb $10.1 \mathrm{gm} / \mathrm{dl}, \mathrm{ESR}-36$ with dimorphic blood picture. TSH was very high (100 ulU/ml) and FT4 was low $0.34 \mathrm{ng} / \mathrm{ml}$. Anti-TG anti-TPO antibodies were positive in high titre. Lipid profile showed elevated cholesterol, triglyceride and LDL-cholesterol. Muscle enzymes were markedly elevated (CPK-12122 U/I and ALT-343 U/L). He was Euglycaemic and S. Creatinine was normal. Serum vitamin D level was low $(19.60 \mathrm{mg} / \mathrm{dl})$, serum Iron level was low normal. Urine routine

1. Brig Gen Md. Abdur Razzak, MBBS, MCPS (Medicine), FCPS (Medicine), APLAR Clinical Fellow in Rheumatology, Chief Physician, CMH, Dhaka (E-mail: razzakprm@gmail.com) 2. Dr Fahd Bin Zia, MBBS, Assistant Registrar of Medicine, AFMC, Dhaka. 
examination revealed no abnormality. ECG (Fig-2) showed evidence of infero lateral ischemia. Troponin I was negative. CXR (Fig-3) showed borderline Cardiomegaly. His ANA was -ve. ENA profile showed only SmD1 positive. Cross limb EMG (Fig-4) showed evidence of myopathy and NCS (Fig-5) revealed motor axonal Polyneuropathy of both upper and lower limbs. Muscle biopsy revealed evidence of inflammatory myopathy (Fig-6). Therefore, he was finally diagnosed as a case of autoimmune primary hypothyroidism. Other associated diagnoses were diastolic hypertension, dyslipidemia, Ischemic heart diseases, inflammatory myopathy, peripheral neuropathy, Vit-D insufficiency, Alopecia uriversalis, depression and dementia. He has been treated with Thyroxin replacement, lipid lowering, antihypertensive and antianginal drugs, Iron, Calcium, Vit-D supplements, physiotherapy and exercise. He was advised for consultation with dermatologist for alopecia universalis. His condition gradually improving and he will be followed up at 1,3 and 6 months.

\section{Discussion}

Hypothyroidism is a common endocrine disease with adverse effects on almost every system of the body. Patients with hypothyroidism are frequently found anemic which might result from bone marrow depression, decreased erythropoietin production, comorbid diseases, or concomitant iron, vitamin B12, or folate deficiency. Altered iron metabolism and oxidative stress may contribute to anemia in hypothyroidism ${ }^{3}$. Modern concepts and approaches suggests that total cholesterol, LDL cholesterol, and possibly triglycerides are increased in patients with subclinical hypothyroidism, whereas high-density lipoprotein (HDL) cholesterol and $L p(a)$ remain unchanged ${ }^{4}$. Hypothyroidism has been recognized as a cause of secondary hypertension. Previous studies have demonstrated elevated blood pressure values in hypothyroid patients. Adequate thyroid hormone replacement therapy successfully reduced blood pressure ${ }^{5}$. Subclinical hypothyroidism is associated with ischemic heart disease and increased cardiovascular mortality. Impaired cardiac muscle relaxation, decreased heart rate, and decreased stroke volume contribute to heart failure in hypothyroidism ${ }^{6}$. Alteration in thyroid status can lead to changes in systolic and diastolic function of left ventricle. Heart is very sensitive to thyroid hormone changes and cardiac disorders are commonly associated with both hyper and hypothyroidism. Dilated cardiomyopathy is a very rare presentation of hypothyroidism ${ }^{7}$. Serous effusions have been thought to be an unusual presentation of hypothyroidism and most commonly have been associated with ascites, pericardial fluid and heart failure. The majority of effusions in patients with hypothyroidism were due to other diseases ${ }^{8}$. Myopathy in Hypothyroidism is usually mild; they include weakness, pain, aching, and stiffness. But sometimes hypothyroid myopathy manifests as polymyositis-like myopathy with proximal muscle weakness and an increased creatine kinase level. In these cases, the hypothyroidism is thought to have predisposed individuals to rhabdomyolysis.
An increase of muscle enzymes in hypothyroidism has sometimes been correlated with a polymyositis-like syndrome and hypothyroid patients have been misdiagnosed and mismanaged as suffering from polymyositis ${ }^{9}$. Peripheral neuropathy may be caused by severe, long-term, untreated hypothyroidism. Signs and symptoms of peripheral neuropathy may include pain, a burning sensation or numbness and tingling in the area affected by the nerve damage. It may also cause muscle weakness or loss of muscle control. Although the association between hypothyroidism and peripheral neuropathy isn't fully understood, it's known that hypothyroidism can cause fluid retention resulting in swollen tissues that exert pressure on peripheral nerves. It may also produce carpal tunnel syndrome and other entrapment neuropathy due to deposition of myxomatous tissue ${ }^{10}$. Severe and prolonged hypothyroidism and hyperthyroidism can cause loss of hair. The loss is diffuse and involves the entire scalp rather than discrete areas. The hair appears uniformly sparse. Regrowth is usual with successful treatment of the thyroid disorder, though it will take several months and may be incomplete. Autoimmune alopecia may also be associated with autoimmune hypothyroidism ${ }^{11}$. The most common cause of hypothyroidism is an autoimmune disorder known as Hashimoto's thyroiditis. Autoimmune disorders occur when your immune system produces antibodies that attack your own tissues. Sometimes this process involves your thyroid gland. Autoimmune thyroid disease is common in lupus. It is believed that about $6 \%$ of people with lupus have hypothyroidism and about $1 \%$ have hyperthyroidism. Hypothyroidism can cause weight gain, fatigue, depression, moodiness, and dry hair and skin2. It's found that 79\% of the 71 men with thyroid problems had ED to some extent. It was more common in men with severe hypothyroidism. Fortunately, treatment for both conditions can relieve erection problems ${ }^{12}$. Hypothyroidism is associated with factors of metabolic syndrome such as dyslipidemia, hypertension, obesity, and often insulin resistance. It has been reported that $95 \%$ of newly diagnosed hypothyroid patients have increased levels of cholesterol and $5 \%$ of them have hypertriglyceridemia ${ }^{13}$. Obstructive sleep apnea (OSA) and hypothyroidism are relatively common disorders that have similar clinical features and are thought to be causally linked.

Obstructive sleep apnea (OSA) is a common disorder in which sleep-disordered breathing causes disrupted sleep as well as other sequelae ${ }^{14}$. While $30 \%$ of the patients with hypothyroidism had NAFLD, $19 \%$ of those having thyroid function levels in the normal thyroid group had a dual diagnosis. These researchers, too, found that the worse the hypothyroidism, the more likely the participants were to have NAFLD ${ }^{1}$. Although they're separate diseases, depression is sometimes a symptom of hypothyroidism. That's when your thyroid gland doesn't make enough thyroid hormone. Medication can boost those levels, and that can improve or get rid of your symptoms, including depression. All of that may cause you to feel depressed. Thyroid disease can affect your mood-primarily causing either anxiety or depression. Generally, the more severe the thyroid disease, the more severe the mood changes e.g. 
Anxiety and Irritability. Myxedema (crisis) coma is a loss of brain function as a result of severe, longstanding low level of thyroid hormone in the blood (hypothyroidism). Myxedema coma is considered a rare life-threatening complication of hypothyroidism and represents one of the more serious side effects of of thyroid disease ${ }^{15}$. Apart from the dreaded complication of myxedema coma which involves the central nervous system, hypothyroid patients undergoing surgery are predisposed to anemia, electrophysiological disturbances, and hypotension, all of which can precipitate cardiovascular collapse. Recovery from anesthesia may be delayed. Anesthesia in untreated hypothyroidism may precipitate myxoedema coma ${ }^{16}$.

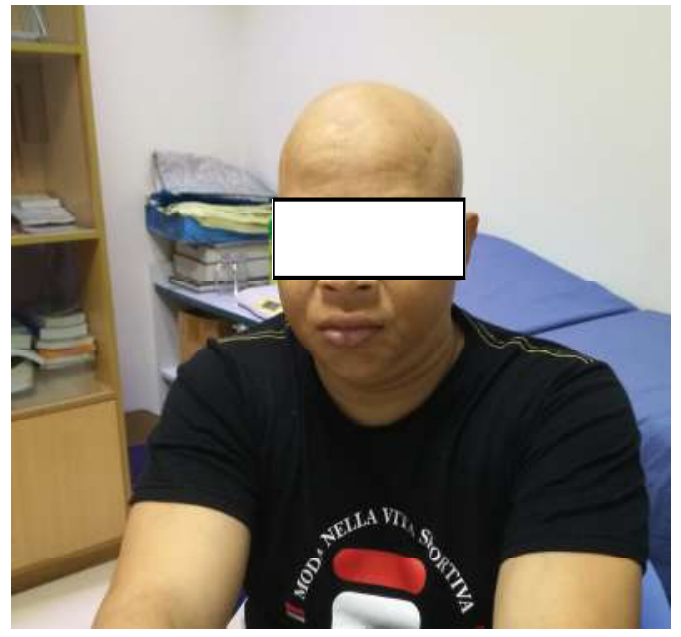

Figure-1: Depressed, dull, puffy face and alopecia universalis.
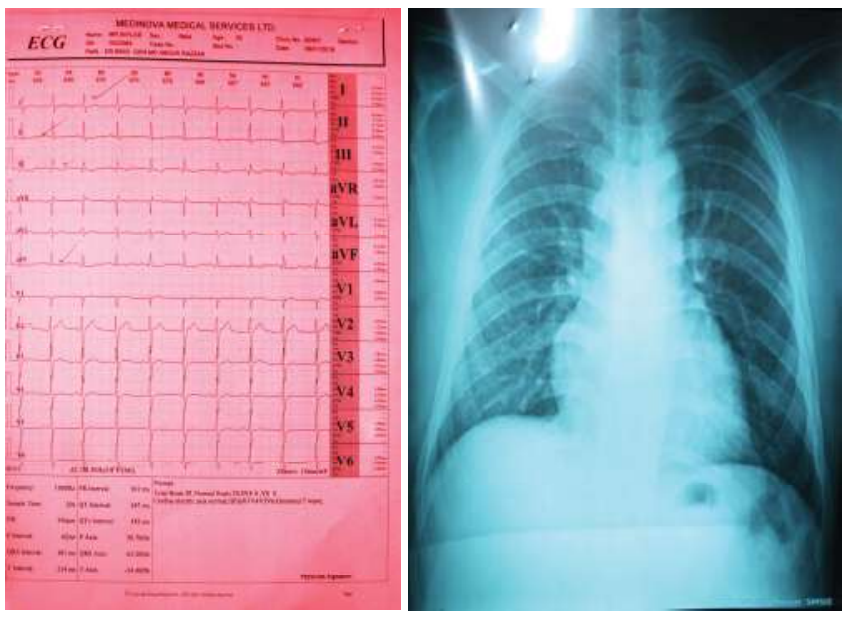

Figure-2: (Inferior Ischemia) Figure-3: CXR (Mild Cardiomegaly)

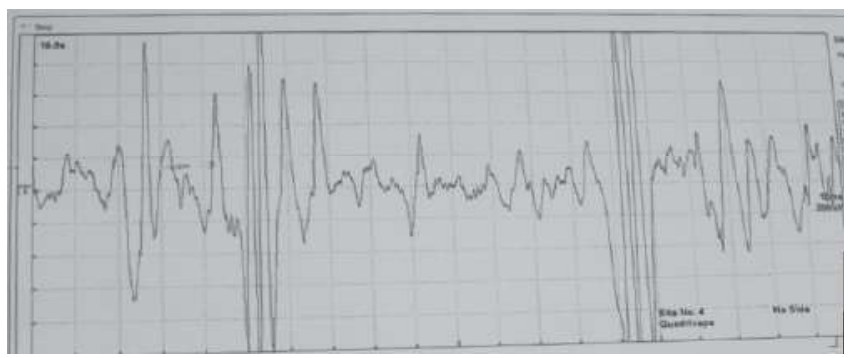

Figure-4: EMG (Inflammatory myopathy)

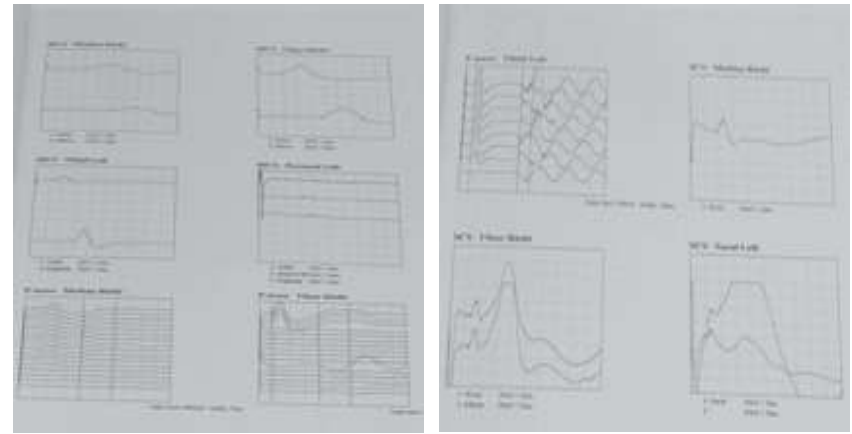

Figure-5: EMG (Inflammatory myopathy)
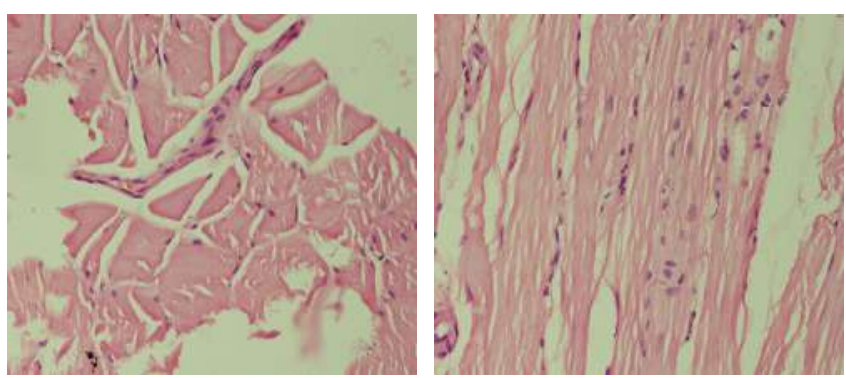

Figure-6: Muscle Biopsy (Inflammatory Myopathy)

\section{Conclusion}

Hypothyroidism is a multi-faceted and multidimensional disease. If the diagnosis is delayed it may produce so many complications. Mortality is due to cardiac, CNS and fetomaternal complications. Autoimmune hypothyroidism is an organ specific autoimmune disease and it may be associated with other autoimmune diseases. It's on of the endocrine causes of obesity and has got link with metabolic syndrome and NAFLD. Initial symptoms of hypothyroidism are nonspecific and vague. High index of suspicion, adequate knowledge and experience is needed for early diagnosis and effective treatment. Timely intervention can improve most of the symptoms and prevent most of the complications. Optimum thyroxine replacement therapy improves the prognosis and patient can lead almost normal life. Regular follow up is essential for better outcome.

\section{References}

1. Mantovani A, Nascimbeni F, Lonardo A et al. Association between Primary Hypothyroidism and Nonalcoholic Fatty Liver Disease: A Systematic Review and Meta-Analysis. Thyroid. 2018; 28(10):1270-84.

2. Lee HJ, Li CW, Hammerstad SS et al. Immunogenetics of autoimmune thyroid diseases: A comprehensive review. J Autoimmun. 2015; 64: 82-90.

3. Antonijević N, Nesović M, Trbojević B et al. Anemia in hypothyroidism. Medicinski Pregled. 1999; 52(3-5):136-40.

4. Neves C, Alves M, Medina JL et al. Thyroid diseases, dyslipidemia and cardiovascular pathology. Rev Port Cardiol. 2008; 27:1211-36.

5. Fletcher AK, Weetman AP. Hypertension and hypothyroidism. Journal of Human Hypertension. 1998; 12:79-82.

6. Razvi S, Shakoor A, Vanderpump M et al. The influence of age on the relationship between subclinical hypothyroidism and 
ischemic heart disease: A metaanalysis. J Clin Endocrinol Metab. 2008; 93(8):2998-3007.

7. Kasper EK, Agema WR, GM Hutchins GM et al. The causes of dilated cardiomyopathy: A clinicopathologic review of 673 consecutive patients. J Am Coll Cardiol. 1994; 23(3):586-90.

8. Gottehrer A, Roa J, Stanford GG et al. Hypothyroidism and pleural effusions. Chest. 1990; 98:1130-2.

9. Madariaga MG, Gamarra N, Dempsey S et al. Polymyostis -like syndrome in hypothyroidism: Review of cases reported over the past twenty-five years. Thyroid. 2002; 12:331-6.

10. Han $\mathrm{C}, \mathrm{He} X, \mathrm{Xia} X$ et al. Subclinical Hypothyroidism and Type 2 Diabetes: A Systematic Review and Meta-Analysis. PLoS ONE. 2015; 10(8): e0135233.

11. Bouscarat F, Prevot MH, Matheron S. Alopecia associated with indinavir therapy. N Engl J Med. 1999; 341(8):618.

12. Thomas R, Reid RL. Thyroid disease and reproductive dysfunction: A review. Obstet Gynecol. 1987; 70(5):789-98.

13. Suh S, Baek J, Bae JC et al. Endocrinology and Metabolism. Endocrinol Metab (Seoul). 2015; 30(1):47-52.

14. Kittle WM, Chaudhary BA. Sleep apnea and hypothyroidism. Southern Medical journal. 1988; 81(11):1421-5.

15. Neal JM, Yuhico RJO. "Myxedema Madness" Associated with Newly Diagnosed Hypothyroidism and Obstructive Sleep Apnea. JCSM. 2012; 8(6):717-8.

16. Polyzos SA, Anastasilakis AD. Clinical complications following thyroid fine-needle biopsy: A systematic review. Clinical Endocrinology. 2009; 71(2):157-65. 